Our study
provides a
rationale for
the mechanism
by which
endothelin
A-receptor
blockers
can reduce
albuminuria

$\Rightarrow$ DIABETIC NEPHROPATHY

\title{
Atrasentan stabilizes the endothelial glycocalyx
}

Endothelin receptor antagonists, such as atrasentan, can reduce albuminuria in patients with diabetic nephropathy. Now, researchers show that atrasentan restores glomerular endothelial glycocalyx coverage. "In the study we not only show that atrasentan restores the glycocalyx in diabetic nephropathy, but also show that this effect depends on downregulation of the enzyme that breaks down the heparan sulphate component of the glycocalyx, heparanase," says researcher Margien Boels.

To examine the mechanisms by which atrasentan confers its antialbuminuric effects, Boels et al. examined the effect of the agent on glycocalyx stability in mice with diabetes. Treatment with atrasentan for 4 weeks reduced progressive albuminuria without any notable change in glomerular capillary size, mesangial area or podocyte number. Endothelial glycocalyx coverage, which was decreased in diabetic mice was, however, restored to normal levels by atrasentan, in association with reduced levels of heparanase. The researchers also observed a shift in the balance of macrophages from proinflammatory M1 macrophages in diabetic mice to regulatory M2 macrophages in atrasentan-treated mice. "Our study provides a rationale for the mechanism by which endothelin A-receptor blockers can reduce albuminuria," says Boels. "We demonstrate that diabetic nephropathy is associated with glomerular inflammation, where macrophages and endothelial cells seem to produce increased levels of heparanase. Administration of atrasentan shifts the balance from M1 to M2 glomerular macrophages and reduces heparanase activity derived from macrophages as well as endothelial cells."

Susan J. Allison

ORIGINAL ARTICLE Boels, M. G. S. et al. Atrasentan reduces albuminuria by restoring the glomerular endothelial glycocalyx barrier in diabetic nephropathy. Diabetes http://dx.doi.org/ $\underline{10.2337 / \mathrm{db} 15-1413}$ 